\title{
Remesas familiares y el COVID-19: eficiencia en los pronósticos para reducir la especulación en la región centroamericana
}

\section{Family Remittances and COVID-19: Efficiency in Forecasting to Reduce Speculation in the Central American Region}

\author{
Luis Rodrigo Asturias Schaub* \\ UNIVERSIDAD DEL ISTMO, GUATEMALA \\ Juan Fernando Díaz Lara* \\ UNIVERSIDAD RAFAEL LANDÍVAR, GUATEMALA
}

\section{Resumen}

La presente investigación aplica un método de pronóstico adecuado a los flujos de remesas que se vieron afectados por el efecto de la crisis generada por el COVID-19 en la región centroamericana, con la intención de que sirva además como un método de referencia para priorizar criterios de eficiencia en las proyecciones de estos flujos de remesas y contribuir de esta forma a reducir la incertidumbre de las autoridades monetarias, de los inversionistas y de los agentes económicos para facilitar el proceso de toma de decisiones.

Al aplicar el modelo de pronóstico sugerido para cada país de la región, se obtuvo un margen de error mínimo, tanto en el valor absoluto en unidades monetarias como en términos relativos, en función de los porcentajes de la diferencia entre la estimación y el valor real que en promedio ascendió a $8.4 \%$ y menos de una desviación estándar.

Adicionalmente, se pretende evidenciar que junto con otras variables macroeconómicas comprometidas ante la situación del COVID-19, las remesas siguen siendo un flujo de ingresos resiliente.

Palabras Clave: remesas familiares, pronóstico, macroeconomía, Centroamérica, ARIMA.

\section{Abstract}

This research applies an adequate forecasting method to the remittance flows that were affected from the crisis generated by COVID-19 in the Central American region, with the aim to provide a reference tool to prioritize forecasting efficiency criteria and thus contribute to reduce the uncertainty of the monetary authorities, the investors and the economic agents, facilitating the decision-making process.

By applying the forecast model suggested for each country in the region, there was a minimum margin of error, both in the absolute value in monetary units and in relative terms as a function of the percentages of the difference between the estimate and the real value that in average amounted to $8.4 \%$ corresponding to less than one standard deviation.

It is intended to show that in addition to other macroeconomic variables compromised by the COVID-19 situation, remittances continue to be a resilient income stream.

Key Words: family remittances, forecast, macroeconomics, Central America, ARIMA.

*lasturias@unis.edu.gt

*fernandoecon@gmail.com 


\section{INTRODUCCIÓN}

os procesos de integración económica normalmente son entendidos como una conjugación de procesos positivos y negativos que, una vez encaminados a través de la obtención de saldos favorables, comienzan a ser rentables mediante la evolución desde áreas de libre comercio hasta convertirse en uniones aduaneras, mercados comunes, uniones económicas y monetarias y finalmente con la consolidación de una unión política. No obstante, en el proceso integrador, existen rasgos comunes que luego de ciertas dinámicas endógenas (como en el caso de la fuerza laboral) pasan a constituir una variable crítica como común denominador de las economías y en donde el pago neto a factores, por ejemplo, comienza a tomar mayor relevancia, siendo este el caso de las remesas familiares.

Con una participación cercana al 10\% del Producto Interno Bruto regional, las remesas familiares representan una faceta externa de los mecanismos de integración de la fuerza laboral centroamericana y reflejan que tácitamente la fuerza laboral regional que trabaja en Estados Unidos está integrada con un objetivo común: mantener un ritmo de envío continuo de flujos de divisas a sus familiares y allegados en su país de origen como producto del trabajo lejos de sus países.

El alcance de las remesas ha pasado a ser muy relevante, siendo ahora considerada como una variable importante con relación al tipo de cambio, el sostenimiento del consumo y de la demanda agregada en general, así como un mecanismo de mitigación de la pobreza y como una forma de contener la elevada desigualdad económica, en donde todos estos factores representan otra cara de la integración regional actual. Por lo tanto, más allá de las especulaciones y de las inferencias con fundamentos escasos, esta investigación pretende poner a disposición una forma adecuada de pronosticar los flujos de remesas a partir de la crisis generada por el COVID-19 en la región centroamericana, demostrando una alta eficacia de predicción en el método y contribuyendo con esto a reducir la pérdida de eficiencia en la toma de decisiones para las autoridades monetarias los países y para los agentes económicos en general. 
Vale la pena mencionar que dentro de la investigación se toma en cuenta a los siguientes países miembros de la región Centroamericana: Guatemala, El Salvador, Honduras, Nicaragua, Costa Rica, Panamá y República Dominicana (por cercanía, características socioeconómicas y comerciales similares al resto de países del istmo) excluyendo a Belice debido a la falta de datos disponibles sobre remesas familiares.

\section{Aspectos Teóricos}

\section{LAS REMESAS Y SU INCIDENCIA FAVORABLE}

Según Larotta (2019), la decisión de migrar y el uso de las remesas como producto de esta movilidad humana son decisiones que los hogares deben tomar de acuerdo con su condición, sus necesidades inmediatas, sus emergencias y planes para el futuro. De esta cuenta las remesas, además de proveer una seguridad monetaria para los hogares, pueden estimular inversiones en capital físico y humano, así como aumentar la flexibilidad de los miembros del hogar en sus roles sociales y su participación en la comunidad.

A pesar de su importancia, la evidencia empírica relacionada con su uso e incidencia en el consumo y la inversión es limitada y poco concluyente. No obstante, debido a su alta participación relativa, el Banco Mundial (2018) sostiene que las remesas tienen un papel preponderante en la reducción de la pobreza de las economías en desarrollo, y en especial para mitigar su incidencia y severidad.

En la misma línea, las remesas no solamente contribuyen a mejorar las condiciones de ingreso y favorecer los principales indicadores de bienestar en los hogares con migrantes, sino que también benefician a los hogares que no reciben remesas y, a la economía en general, mediante el aumento de las demandas derivadas y en la aceleración de los flujos comerciales.

Si bien la mayor parte de beneficios son económicos, las remesas plantean el constante reto de superar otros retos que derivan de condiciones sociales que pueden verse agravadas y que incluyen desintegración familiar, pérdida de identidad cultural, el riesgo asociado a las condiciones del traslado, la trata de personas y la pérdida de talentos en los países de origen, entre otras. 


\section{LA MIGRACIÓN, LAS REMESAS Y LA INTEGRACIÓN}

De acuerdo con Álvarez (2012), el aumento de la migración de centroamericanos hacia Estados Unidos y el incremento derivado en el flujo de remesas hacia los países de origen ha contribuido a que la región centroamericana evidencia una serie de efectos, financieros, que constituyen elementos integradores por la alta dependencia que han comenzado a generar en las economías de los países del istmo.

Por lo tanto, las remesas evidencian una alta incidencia en el turismo, las telecomunicaciones, el transporte, el comercio nostálgico y las mismas transferencias que provienen del exterior (Álvarez, 2012). A esto, debe sumarse que los migrantes establecen redes de apoyo en el país de destino y que fomentan redes de apoyo que inciden favorablemente en el capital social, en una alta escala para las personas en el exterior y con un impacto relativamente mayor en los grupos familiares en el país de origen (Organización Internacional para las Migraciones, 2018).

En todo caso, las fluctuaciones en los flujos de remesas provocan distorsiones (positivas y negativas) en el tipo de cambio, en los niveles agregados de consumo, en la inversión y la dinámica de ciertas actividades del sector secundario, especialmente la construcción (Consejo Monetario Centroamericano, 2018), evidenciando así que la región centroamericana tiene como uno de sus denominadores comunes, es decir, como uno de sus factores integradores comunes, el flujo de remesas internacionales. Por lo tanto, es necesario contar con mecanismos de predicción eficientes para minimizar la incertidumbre y cuantificar la magnitud de las variaciones de este flujo de divisas en aras de mejorar los procesos de toma decisiones tanto a nivel macroeconómico como microeconómico dentro de los países del Istmo. Vale la pena mencionar que dentro del modelo se consideran a los países: Guatemala, El Salvador, Honduras, Nicaragua, Costa Rica, Panamá y República Dominicana (por cercanía y características socioeconómicas y comerciales similares al resto de países del istmo) excluyendo a Belice debido a la falta de datos de remesas familiares. 


\section{Metodología}

Teniendo en cuenta que el presente estudio se centra en contar con una metodología adecuada para pronosticar el flujo de remesas a la región centroamericana con base a los efectos del COVID-19, se realiza a continuación una descripción del método empleado y derivado de Box \& Jenkins (1978), técnicamente conocido como metodología ARIMA.

Partiendo de la filosofía de que los datos deben de hablar por sí mismos, el método propuesto por Box \& Jenkins es identificar y estimar un modelo estadístico que se interprete como generador de los datos muestrales. Para pronosticar los flujos de remesas en la región con este modelo, debe suponerse que sus características son constantes a través del tiempo y, en particular, en periodos futuros. Así, la razón para requerir datos estacionarios es que todo modelo que se infiera a partir de ellos puede interpretarse como estacionario o estable en sí mismo, y proporcione, por consiguiente, una base válida para pronosticar (Pokorny, 1987).

Varios de los modelos estadísticos utilizados para realizar proyecciones en el campo económico consideran tener medias y varianzas constantes con autocovarianzas que no dependen del tiempo sino sólo de los retardos. No obstante, en las series de tiempo económicas, y en lo que se refiere por lo tanto a las remesas, la mayoría de los casos en los países analizados no se comportan de forma estacionaria, ya sea porque suelen ir cambiando de nivel en el tiempo o porque la varianza no es constante. A este tipo de procesos se les considera procesos integrados (Villavicencio, 2014).

Las series de tiempo que presentan los flujos de remesas se deben diferenciar $d$ veces para hacerlas estacionarias y luego aplicarles un modelo $\operatorname{ARIMA}(p, d, q)$, es decir, una serie de tiempo autoregresiva integrada de media móvil. Donde $p$ denota el número de términos autoregresivos, $d$ el número de veces que la serie debe ser diferenciada para hacerla estacionaria y, $q$ el número de términos de la media móvil invertible (Villavicencio, 2014).

$$
Y_{t}^{d}=c+\phi_{1} Y_{t-1}^{d}+\cdots+\phi_{p} Y_{t-p}^{d}+a_{t}^{d}+\theta_{1} a_{t-1}^{d}+\theta_{q} a_{t-q}^{d}
$$


Según Villavicencio (2014) la construcción de los modelos $\operatorname{ARIMA}(p, d, q)$, se lleva de manera iterativa mediante un proceso en el que se puede distinguir cuatro etapas:

- Identificación. Utilizando los datos ordenados cronológicamente se intentará sugerir un modelo $\operatorname{ARIMA}(p, d, q)$ que merezca la pena ser investigada. El objetivo es determinar los valores $p, d, q$ es que sean apropiados para reproducir la serie de tiempo. En esta etapa es posible identificar más de un modelo candidato que pueda describir la serie.

- Estimación. Considerando el modelo apropiado para la serie de tiempo se realiza inferencia sobre los parámetros.

- Validación. Se realizan contraste de diagnóstico para validar i el modelo seleccionado se ajusta a los datos, si no es así, escoger el próximo modelo candidato y repetir los pasos anteriores.

- Predicción. Seleccionado el mejor modelo $\operatorname{ARIMA}(p, d, q)$ se pueden hacer pronósticos en términos probabilísticos de los valores futuros.

Esta metodología basa la selección de un modelo en forma iterativa, debido a que en cada etapa se plantea la posibilidad de rehacer las etapas previas, y en el de parametrización escueta, también denominado parsimonia, considerando que se trata de proponer un modelo capaz de representar la serie con el mínimo de parámetros posibles y únicamente acudir a una ampliación del mismo en caso de que sea estrictamente necesario para describir el comportamiento de la serie.

\section{Modelos ARIMA estacionales SARIMA $(P, D, Q)(P, D, Q)$}

Para las observaciones registradas en el flujo de remesas, como una serie de tiempo que incluye intervalos de observación menores a un año, es frecuente que presenten variaciones o patrones sistemáticos cada cierto periodo y que sean inferiores a un ańo (las remesas se reportan normalmente de forma mensual). Por lo tanto, a la hora de considerar una estructura ARIMA, deben de captarse además 
los llamados factores estacionales (Villavicencio, 2014). Este tipo de procesos tiene las siguientes características:

- Contiene un componente ARIMA $(p, d, q)$ que modela la dependencia regular, que es la dependencia asociada a observaciones consecutivas.

- Contiene un componente ARIMA $(P, D, Q)$ que modela la dependencia estacional, que está asociada a observaciones separadas por $\mathrm{S}$ periodos.

- La estructura general de un modelo $\operatorname{ARIMA}(p, d, q)$ $(P, D, Q)$

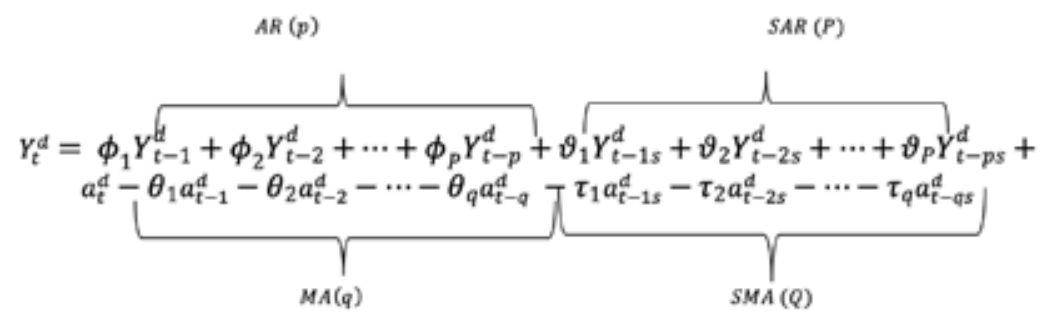

Los parámetros son $\phi_{1}, \ldots \phi_{p}, \theta_{1} \ldots, \theta_{p}, \theta_{1}, \ldots . \theta_{p}, \tau_{1}, \ldots, \tau_{p} y a_{t} \sim N\left(0, \sigma^{2}\right)$.

En donde:

$\underline{Y_{t}^{d}}:$ Pronóstico del flujos mensuales de remesas familiares.

$a_{t}^{d}$ : Innovación diferenciada (componente aleatorio).

$\overline{\phi_{P} Y_{t-p}^{d}:}$ Autorregresivo diferenciado: Valor presente de las remesas familiares que se representa en función de su propio pasado con $p$ resagos.

$\vartheta_{P} Y_{t-p s}^{d}: \quad$ Autorregresivo estacional diferenciado: Valor autorregresivo estacional de las remesas familiares que se representa en función de su propio pasado con $p^{S}$ rezagos.

$\theta_{q} a_{t-q}^{d}:$ Media móvil diferenciado: Valor presente de las remesas familiares en función de todas las innovaciones presentes y pasadas (componente aleatorio) con $q$ rezagos. 
$\overline{\tau_{q} a_{t-q s}^{d}:}$ Media móvil estacional diferenciado: Valor de media móvil estacional de las remesas familiares en función de todas las innovaciones presentes y pasadas (componente aleatorio) $q^{s}$ rezagos.

En sintonía de los diferentes modelos SARIMA utilizados en la investigación de cada país, la cantidad de rezagos utilizados en cada uno es diferente.

La metodología para construir el modelo ARIMA $(p, d, q)$ $(P, D, Q)$ s apropiado para la serie estacional $Y_{1}, Y_{2}, \ldots Y t_{\text {consta }}$ de las fases consideradas en la ilustración número uno las cuales son: Identificación, Estimación, Validación y Predicción (González Casimiro, 2009).

Para el caso específico de las remesas $(Y t)$ se trata de proponer los modelos ARIMA $(p, d, q)(P, D, Q)$ s que puedan representar la evolución de la serie. En primer lugar, se analiza la estacionariedad de los flujos de remesas tanto en varianza como en media y, después, para la serie original del flujo de remesas o aquella transformación estacionaria de la misma, seleccionándose luego los órdenes $(p, d)$ de la estructura regular estacionaria y $(P, D)$ s de la estructura estacional estacionaria. Para esto, se consideran además los siguientes análisis:

Análisis de estacionariedad: Para estudiar la estacionariedad en varianza de la serie del flujo de remesas es un gráfico que permite observar si la variabilidad de la serie es homogénea a lo largo del tiempo. En las series con comportamiento estacional suele suceder que la variabilidad o amplitud del ciclo estacional crece con la tendencia, como en este caso, añadiéndose luego la función de autocorrelación y los contrastes de raíces unitarias (prueba DickeyFuller).

Selección de los órdenes $(p, d)$ y $(P, D)$ : Comprende la elección de los modelos apropiados para la serie estacionaria que se realizan estudiando su función de autocorrelación simple y parcial. Asimismo, se considera: la estimación que se deriva del proceso de identificación; la validación, que trata de comprobar de que el modelo propuesto para proyectar los flujos de remesas se ajuste bien a los datos y reproduzca la estructura de comportamiento de 
la serie (considerando el criterio de Akaike (AIC); la predicción, en donde una vez completa la validación de modelo corroborando la existencia de ruido blanco y el ajuste correcto de los datos es posible generar una predicción para los periodos necesarios.

\section{Resultados}

Considerando los valores presentados por los distintos Bancos Centrales dentro del Consejo Monetario Centroamericano (2020), el ingreso de las remesas familiares en la región centroamericana durante el periodo 2013 al 2019, ha representado en promedio el $10 \%$ del Producto Interno Bruto, y en países como Guatemala, Honduras y El Salvador ha representado hasta el 14\%. El ingreso de las remesas es cada vez mayor, a continuación, se presentan los diferentes resultados específicos para cada uno de los países de Centroamérica incluyendo República Dominicana.

Tabla No. 1 Ingresos por Remesas familiares anuales como PORCENTAJE DEL PIB

\begin{tabular}{|l|c|c|c|c|c|c|c|c|}
\hline Año & $\begin{array}{c}\text { Costa } \\
\text { Rica }\end{array}$ & $\begin{array}{c}\text { El } \\
\text { Salvador }\end{array}$ & Guatemala & Honduras & Nicaragua & $\begin{array}{c}\text { República } \\
\text { Dominicana }\end{array}$ & Panamá & Promedio \\
\hline $\mathbf{2 0 1 3}$ & 1.13 & 17.94 & 9.84 & 16.72 & 9.81 & 6.8 & 0.93 & 9.0 \\
\hline $\mathbf{2 0 1 4}$ & 1.1 & 18.32 & 9.79 & 17.4 & 9.56 & 6.8 & 1.31 & 9.2 \\
\hline $\mathbf{2 0 1 5}$ & 0.94 & 18.16 & 10.3 & 17.77 & 9.36 & 6.96 & 0.87 & 9.2 \\
\hline $\mathbf{2 0 1 6}$ & 0.9 & 18.78 & 11.01 & 18.18 & 9.52 & 6.94 & 0.74 & 9.4 \\
\hline $\mathbf{2 0 1 7}$ & 0.91 & 19.96 & 11.6 & 19.18 & 10.09 & 7.39 & 0.71 & 10.0 \\
\hline $\mathbf{2 0 1 8}$ & 0.82 & 20.64 & 12.76 & 20.33 & 11.49 & 7.59 & 0.7 & 10.6 \\
\hline $\mathbf{2 0 1 9}$ & 0.84 & 20.9 & 13.75 & 22.01 & $\begin{array}{c}\text { No } \\
\text { disponible }\end{array}$ & 7.97 & 0.74 & 11.0 \\
\hline
\end{tabular}

Fuente: Elaboración propia con base en Consejo Monetario Centroamericano.

En el período de enero a mayo de 2020, se evidenció una desaceleración en el flujo de divisas por concepto de remesas en la región centroamericana, considerando el inicio y la acentuación de los efectos derivados de la pandemia provocada por el COVID-19.

Debido a la importancia relativa de las remesas en los países de la región, los efectos han sido disímiles, aunque todos significativos. Por ejemplo, en Guatemala, el mes de abril presentó 
una desaceleración del 20\% y ante esa caída significativa, se hace cada vez más necesario contar con instrumentos de pronóstico que permitan proyectar de mejor forma el comportamiento de la variable ante un clima de incertidumbre. El modelo pronosticado que mejor que puede definir al comportamiento futuro de las remesas es el modelo SARIMA $(1,1,1)(1,0,0)$. A partir de la Tabla No. 2 es posible identificar distintos valores, tomando en cuenta pronóstico altos y bajos con diferentes niveles de confianza, proveyendo así escenarios adecuados para mejorar la estimación.

Tabla No. 2 Remesas familiares en Guatemala de acuerdo a MODELO SARIMA $(1,1,1)(1,0,0)$

- Millones de US\$-

\begin{tabular}{|c|c|c|c|c|c|}
\cline { 2 - 6 } \multicolumn{1}{c|}{} & $\begin{array}{c}\text { Promedio del } \\
\text { Pronóstico de } \\
\text { Remesas }\end{array}$ & $\begin{array}{c}\text { Pronóstico Bajo } \\
\text { con 80\% de } \\
\text { Confianza }\end{array}$ & $\begin{array}{c}\text { Pronóstico } \\
\text { Alto con 80\% } \\
\text { de Confianza }\end{array}$ & $\begin{array}{c}\text { Pronóstico } \\
\text { Bajo con 95\% } \\
\text { de Confianza }\end{array}$ & $\begin{array}{c}\text { Pronóstico Alto } \\
\text { con 95\% de } \\
\text { Confianza }\end{array}$ \\
\hline ago-20 & $1,047.5$ & 975.2 & $1,119.9$ & 936.9 & $1,158.2$ \\
\hline sep-20 & 960.0 & 875.6 & $1,044.4$ & 831.0 & $1,089.0$ \\
\hline oct-20 & $1,022.4$ & 933.0 & $1,111.7$ & 885.7 & $1,159.0$ \\
\hline nov-20 & 891.0 & 798.8 & 983.1 & 750.0 & $1,031.9$ \\
\hline dic-20 & 972.2 & 878.0 & $1,066.3$ & 828.1 & $1,116.2$ \\
\hline ene-21 & 896.8 & 801.0 & 992.7 & 750.3 & $1,043.4$ \\
\hline feb-21 & 877.7 & 780.3 & 975.0 & 728.8 & $1,026.5$ \\
\hline mar-21 & 834.7 & 736.0 & 933.5 & 683.7 & 985.8 \\
\hline abr-21 & 795.6 & 695.5 & 895.7 & 642.5 & 948.8 \\
\hline may-21 & 897.1 & 795.7 & 998.6 & 741.9 & $1,052.3$ \\
\hline jun-21 & 985.1 & 882.3 & $1,087.9$ & 827.9 & $1,142.3$ \\
\hline jul-21 & $1,065.4$ & 961.3 & $1,169.5$ & 906.2 & $1,224.6$ \\
\hline
\end{tabular}

Fuente: Elaboración propia. 
Gráfico No. 1 Pronóstico remesas familiares en Guatemala.

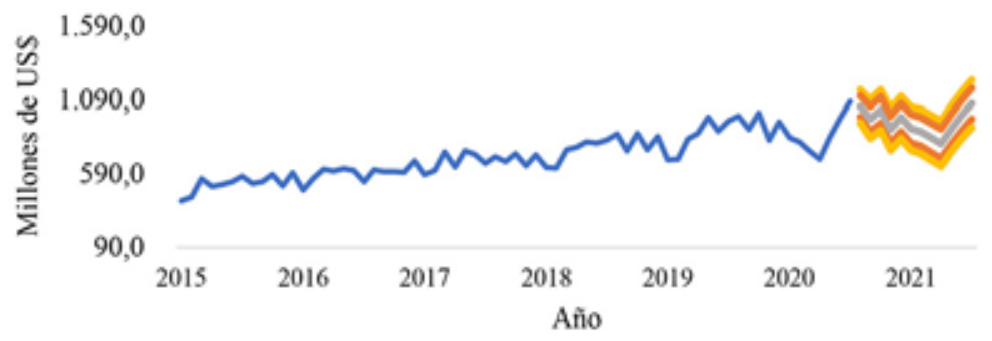

- Valor - Bajo $80 \longrightarrow$ Pronóstico $=$ Alto $95-$ Bajo $95-$ Alto 80

Fuente: Elaboración propia.

El Salvador en el mes abril que tuvo un mayor impacto la crisis provocada por el COVID-19 con una disminución del $40 \%$ en el ingreso de remesas en comparación con el mismo mes del 2019. El mejor modelo para El Salvador es el modelo SARIMA $(0,1,2)$ $(1,0,0)$.

Tabla No. 3 Remesas familiares en El Salvador pronóstico ACUERdo A MODELO SARIMA $(0,1,2)(1,0,0)$

- Millones de US\$ -

\begin{tabular}{|c|c|c|c|c|c|}
\cline { 2 - 6 } \multicolumn{1}{c|}{} & $\begin{array}{c}\text { Promedio del } \\
\text { Pronostico de } \\
\text { Remesas }\end{array}$ & $\begin{array}{c}\text { Pronostico } \\
\text { Bajo con 80\% } \\
\text { de Confianza }\end{array}$ & $\begin{array}{c}\text { Pronostico } \\
\text { Alto con 80\% } \\
\text { de Confianza }\end{array}$ & $\begin{array}{c}\text { Pronostico } \\
\text { Bajo con 95\% } \\
\text { de Confianza }\end{array}$ & $\begin{array}{c}\text { Pronostico Alto } \\
\text { con 95\% de } \\
\text { Confianza }\end{array}$ \\
\hline ago-20 & 496.3 & 455.7 & 536.8 & 434.2 & 558.3 \\
\hline sep-20 & 464.3 & 417.9 & 510.8 & 393.3 & 535.4 \\
\hline oct-20 & 483.9 & 437.2 & 530.6 & 412.5 & 555.3 \\
\hline nov-20 & 463.8 & 416.8 & 510.7 & 392.0 & 535.6 \\
\hline dic-20 & 539.0 & 491.7 & 586.2 & 466.8 & 611.2 \\
\hline ene-21 & 436.8 & 389.3 & 484.3 & 364.2 & 509.4 \\
\hline feb-21 & 457.0 & 409.3 & 504.8 & 384.0 & 530.0 \\
\hline mar-21 & 449.1 & 401.1 & 497.0 & 375.7 & 522.4 \\
\hline abr-21 & 326.5 & 278.2 & 374.7 & 252.7 & 400.2 \\
\hline may-21 & 428.7 & 380.2 & 477.2 & 354.5 & 502.9 \\
\hline jun-21 & 504.3 & 455.5 & 553.0 & 429.7 & 578.8 \\
\hline jul-21 & 540.4 & 491.4 & 589.4 & 465.5 & 615.3 \\
\hline
\end{tabular}

Fuente: Elaboración propia. 
Gráfico No. 2 Pronóstico remesas familiares en El Salvador.

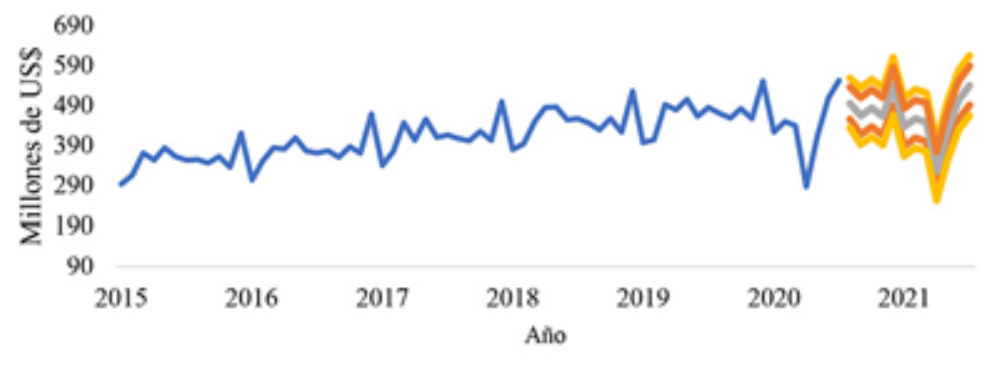

—Valor —Bajo 80 —Pronóstico —Alto 95 - Bajo 95 —Alto 80

Fuente: Elaboración propia.

Del mismo modo, que Guatemala y El Salvador, Honduras tuvo una contracción considerable del $28 \%$ de las remesas en el mes de abril. Con el propósito de generar resultados estables para el pronóstico de Honduras, el mejor modelo según su trayectoria es el modelo SARIMA $(1,1,1)(1,0,0)$.

Tabla No. 4 Remesas familiares en Honduras pronóstico ACUERdo A MOdelo SARIMA $(1,1,1)(1,0,0)$ - Millones de US $\$$ -

\begin{tabular}{|c|c|c|c|c|c|}
\cline { 2 - 6 } \multicolumn{1}{c|}{} & $\begin{array}{c}\text { Promedio del } \\
\text { Pronóstico de } \\
\text { Remesas }\end{array}$ & $\begin{array}{c}\text { Pronostico } \\
\text { Bajo con 80 \% } \\
\text { de Confianza }\end{array}$ & $\begin{array}{c}\text { Pronostico } \\
\text { Alto con 80 \% } \\
\text { de Confianza }\end{array}$ & $\begin{array}{c}\text { Pronostico } \\
\text { Bajo con 95 \% } \\
\text { de Confianza }\end{array}$ & $\begin{array}{c}\text { Pronostico } \\
\text { Alto con 95 \% } \\
\text { de Confianza }\end{array}$ \\
\hline jul-20 & 512.13 & 471.25 & 553.00 & 449.61 & 574.64 \\
\hline ago-20 & 484.60 & 438.31 & 530.89 & 413.81 & 555.40 \\
\hline sep-20 & 480.19 & 431.82 & 528.56 & 406.22 & 554.16 \\
\hline oct-20 & 485.95 & 436.36 & 535.54 & 410.11 & 561.79 \\
\hline nov-20 & 461.91 & 411.38 & 512.44 & 384.63 & 539.19 \\
\hline dic-20 & 483.79 & 432.43 & 535.15 & 405.25 & 562.34 \\
\hline ene-21 & 439.82 & 387.68 & 491.96 & 360.07 & 519.56 \\
\hline feb-21 & 445.57 & 392.67 & 498.47 & 364.66 & 526.47 \\
\hline mar-21 & 410.92 & 357.28 & 464.56 & 328.88 & 492.96 \\
\hline abr-21 & 391.25 & 336.88 & 445.62 & 308.10 & 474.41 \\
\hline may-21 & 437.14 & 382.05 & 492.23 & 352.89 & 521.39 \\
\hline jun-21 & 481.59 & 425.79 & 537.39 & 396.25 & 566.93 \\
\hline
\end{tabular}

Fuente: Elaboración propia. 
Gráfico No. 3 Pronóstico remesas familiares en Honduras.

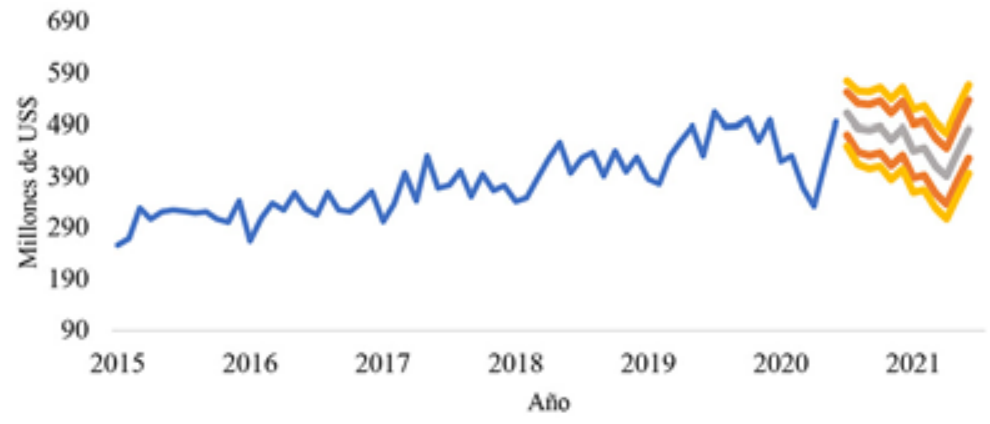

—Valor —Bajo 80 -Pronóstico - Alto $95-$ Bajo 95 - Alto 80 Fuente: Elaboración propia.

Para el caso de Nicaragua es necesario comentar que, desde el segundo semestre del ańo 2019, se han dejado de publicar los datos dentro del Consejo Monetario Centroamericano, el cual es la base de los datos para la presente investigación. Sin embargo, considerando la evolución histórica de los datos se ha determinado que el mejor modelo esta dado por el componente $\operatorname{SARIMA}(0,1,1)(1,1,0)$. En el caso de Nicaragua se han pronosticado tanto los datos restantes del año 2019 como los consecutivos.

Tabla No. 5 Remesas familiares en Nicaragua pronóstico ACUERDO A MODELO SARIMA $(0,1,1)(1,1,0)$

- Millones de US\$

\begin{tabular}{|c|c|c|c|c|c|}
\hline & $\begin{array}{c}\text { Promedio } \\
\text { del } \\
\text { Pronostico } \\
\text { de Remesas }\end{array}$ & $\begin{array}{c}\text { Pronostico } \\
\text { Bajo con } \\
80 \% \text { de } \\
\text { Confianza }\end{array}$ & $\begin{array}{c}\text { Pronostico } \\
\text { Alto con } \\
80 \% \text { de } \\
\text { Confianza }\end{array}$ & $\begin{array}{c}\text { Pronostico } \\
\text { Bajo con } \\
95 \% \text { de } \\
\text { Confianza }\end{array}$ & $\begin{array}{c}\text { Pronostico } \\
\text { Alto con 95\% } \\
\text { de Confianza }\end{array}$ \\
\hline jul-19 & 142.16 & 137.23 & 147.09 & 134.62 & 149.70 \\
\hline ago-19 & 141.78 & 136.66 & 146.89 & 133.95 & 149.60 \\
\hline sep-19 & 135.15 & 129.86 & 140.45 & 127.05 & 143.26 \\
\hline oct-19 & 141.42 & 135.95 & 146.89 & 133.05 & 149.79 \\
\hline nov-19 & 138.54 & 132.90 & 144.18 & 129.91 & 147.17 \\
\hline dic-19 & 160.52 & 154.72 & 166.33 & 151.64 & 169.40 \\
\hline ene-20 & 136.16 & 130.19 & 142.12 & 127.03 & 145.28 \\
\hline feb-20 & 137.94 & 131.82 & 144.06 & 128.58 & 147.30 \\
\hline
\end{tabular}




\begin{tabular}{|c|c|c|c|c|c|}
\cline { 2 - 6 } \multicolumn{1}{c|}{} & $\begin{array}{c}\text { Promedio del } \\
\text { Pronóstico de } \\
\text { Remesas }\end{array}$ & $\begin{array}{c}\text { Pronostico } \\
\text { Bajo con 80\% } \\
\text { de Confianza }\end{array}$ & $\begin{array}{c}\text { Pronostico } \\
\text { Alto con 80\% } \\
\text { de Confianza }\end{array}$ & $\begin{array}{c}\text { Pronostico } \\
\text { Bajo con 95\% } \\
\text { de Confianza }\end{array}$ & $\begin{array}{c}\text { Pronostico Alto } \\
\text { con 95\% de } \\
\text { Confianza }\end{array}$ \\
\hline mar-20 & 147.76 & 141.49 & 154.03 & 138.17 & 157.35 \\
\hline abr-20 & 150.01 & 143.59 & 156.43 & 140.19 & 159.83 \\
\hline may-20 & 152.46 & 145.90 & 159.03 & 142.42 & 162.50 \\
\hline jun-20 & 141.39 & 134.69 & 148.10 & 131.14 & 151.65 \\
\hline jul-20 & 150.09 & 142.64 & 157.53 & 138.71 & 161.47 \\
\hline ago-20 & 151.38 & 143.70 & 159.06 & 139.64 & 163.12 \\
\hline sep-20 & 145.77 & 137.86 & 153.67 & 133.68 & 157.86 \\
\hline oct-20 & 152.62 & 144.49 & 160.74 & 140.18 & 165.05 \\
\hline nov-20 & 149.35 & 141.01 & 157.69 & 136.59 & 162.11 \\
\hline dic-20 & 172.65 & 164.10 & 181.21 & 159.57 & 185.74 \\
\hline ene-21 & 147.70 & 138.94 & 156.46 & 134.31 & 161.10 \\
\hline feb-21 & 147.19 & 138.23 & 156.15 & 133.48 & 160.89 \\
\hline mar-21 & 160.24 & 151.08 & 169.40 & 146.23 & 174.25 \\
\hline abr-21 & 159.34 & 149.99 & 168.69 & 145.04 & 173.64 \\
\hline may-21 & 166.19 & 156.65 & 175.73 & 151.60 & 180.78 \\
\hline jun-21 & 155.97 & 146.25 & 165.70 & 141.10 & 170.84 \\
\hline & & & & & \\
\hline
\end{tabular}

Fuente: Elaboración propia.

Gráfico No. 4 Pronóstico remesas familiares en Nicaragua.

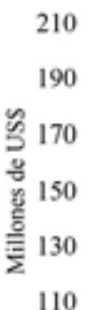

110

90

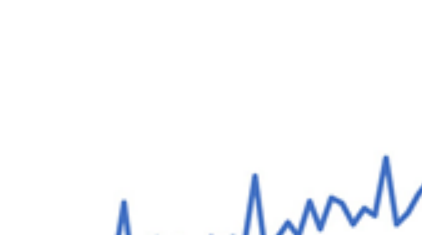

MNMNV

$\begin{array}{lllllll}2015 & 2016 & 2017 & 2018 & 2019 & 2020 & 2021\end{array}$

Año

— Valor —Bajo 80 - Pronóstico - Alto 95 - Bajo 95 - Alto 80

Fuente: Elaboración propia.

República Dominicana presenta similitudes comparadas con Guatemala, Honduras y El Salvador considerando que el mes que presentó una contracción considerable fue también el mes de abril 
con una disminución en el ingreso de las remases del 32\%. Por lo que el mejor modelo que pronostica y simula el comportamiento de las mismas el modelo SARIMA $(0,1,4)(1,0,0)$.

Tabla No. 6 Remesas familiares en República Dominicana PRONÓSTICO ACUERDO A MODELO SARIMA $(0,1,4)(1,0,0)$

- Millones de US\$ -

\begin{tabular}{|c|c|c|c|c|c|}
\cline { 2 - 6 } \multicolumn{1}{c|}{} & $\begin{array}{c}\text { Promedio del } \\
\text { Pronóstico de } \\
\text { Remesas }\end{array}$ & $\begin{array}{c}\text { Pronostico } \\
\text { Bajo con 80\% } \\
\text { de Confianza }\end{array}$ & $\begin{array}{c}\text { Pronostico } \\
\text { Alto con 80\% } \\
\text { de Confianza }\end{array}$ & $\begin{array}{c}\text { Pronostico } \\
\text { Bajo con 95\% } \\
\text { de Confianza }\end{array}$ & $\begin{array}{c}\text { Pronostico Alto } \\
\text { con 95\% de } \\
\text { Confianza }\end{array}$ \\
\hline ago-20 & 615.38 & 530.09 & 650.68 & 498.17 & 782.60 \\
\hline sep-20 & 561.95 & 500.53 & 623.37 & 468.01 & 655.88 \\
\hline oct-20 & 544.12 & 482.45 & 605.79 & 449.80 & 638.44 \\
\hline nov-20 & 587.32 & 519.93 & 654.71 & 484.26 & 690.39 \\
\hline dic-20 & 650.03 & 582.08 & 717.99 & 546.11 & 753.96 \\
\hline ene-21 & 606.09 & 537.57 & 674.60 & 501.31 & 710.87 \\
\hline feb-21 & 611.94 & 542.88 & 681.01 & 506.31 & 717.57 \\
\hline mar-21 & 564.03 & 494.41 & 633.64 & 457.56 & 670.50 \\
\hline abr-21 & 485.27 & 415.10 & 555.43 & 377.96 & 592.57 \\
\hline may-21 & 638.70 & 568.00 & 709.40 & 530.57 & 746.83 \\
\hline jun-21 & 701.16 & 629.92 & 772.39 & 592.21 & 810.11 \\
\hline jul-21 & 757.69 & 685.92 & 829.47 & 647.93 & 867.46 \\
\hline
\end{tabular}

Fuente: Elaboración propia.

Gráfico No. 5 Pronóstico remesas familiares en República DOMINICANA.

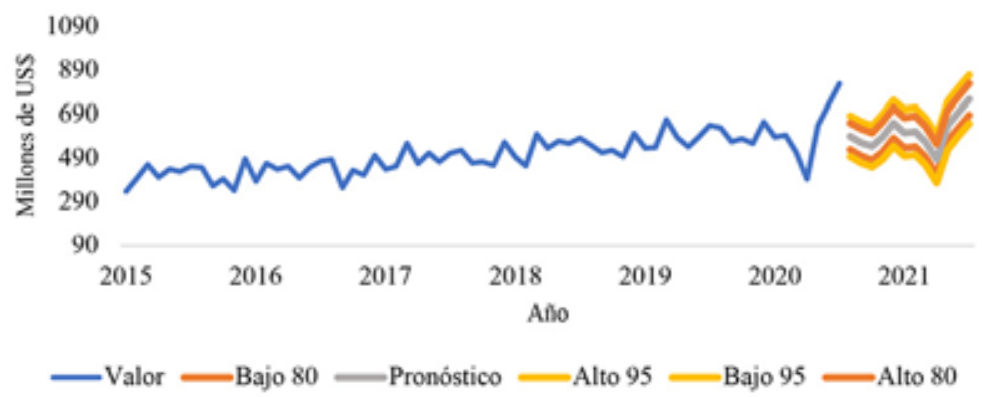

Fuente: Elaboración propia. 
Los pronósticos correspondientes a Costa Rica y Panamá se encuentran representados en trimestres tomando en cuenta la forma en que los publican. Es necesario señalar que tanto Costa Rica como Panamá debido a sus características particulares son los países de Centroamérica que menos remesas reciben del exterior, con un 0.84\% del PIB para Costa Rica y un $0.74 \%$ para Panamá.

Para Costa Rica el mejor modelo que se aproxima a la tendencia en el ingreso de las remesas familiares es el modelo SAR $(1,1,0)$.

Tabla No. 7 Remesas familiares en Costa Rica pronóstico ACUERDO A MODELO SAR $(0,0,0)(1,1,0)$

- Millones DE US\$ -

\begin{tabular}{|l|c|c|c|c|c|}
\cline { 2 - 6 } & $\begin{array}{c}\text { Promedio del } \\
\text { Pronóstico de } \\
\text { Remesas }\end{array}$ & $\begin{array}{c}\text { Pronóstico } \\
\text { Bajo con 80\% } \\
\text { de Confianza }\end{array}$ & $\begin{array}{c}\text { Pronóstico } \\
\text { Alto con 80\% } \\
\text { de Confianza }\end{array}$ & $\begin{array}{c}\text { Pronóstico } \\
\text { Bajo con 95\% } \\
\text { de Confianza }\end{array}$ & $\begin{array}{c}\text { Pronostico } \\
\text { Alto con 95\% } \\
\text { de Confianza }\end{array}$ \\
\hline II Trim. 2020 & 126.5 & 121.0 & 132.0 & 118.1 & 135.0 \\
\hline III Trim.2020 & 132.1 & 126.6 & 137.6 & 123.7 & 140.5 \\
\hline IV Trim. 2020 & 132.1 & 126.6 & 137.6 & 123.7 & 140.5 \\
\hline I Trim. 2021 & 114.9 & 109.4 & 120.4 & 106.5 & 123.3 \\
\hline II Trim.2021 & 128.8 & 123.1 & 134.5 & 120.1 & 137.5 \\
\hline III Trim. 2021 & 134.3 & 128.6 & 139.9 & 125.6 & 142.9 \\
\hline IV Trim. 2021 & 134.6 & 128.9 & 140.2 & 125.9 & 143.3 \\
\hline
\end{tabular}

Fuente: Elaboración propia.

Gráfico No. 6 Pronóstico remesas familiares en Costa Rica.

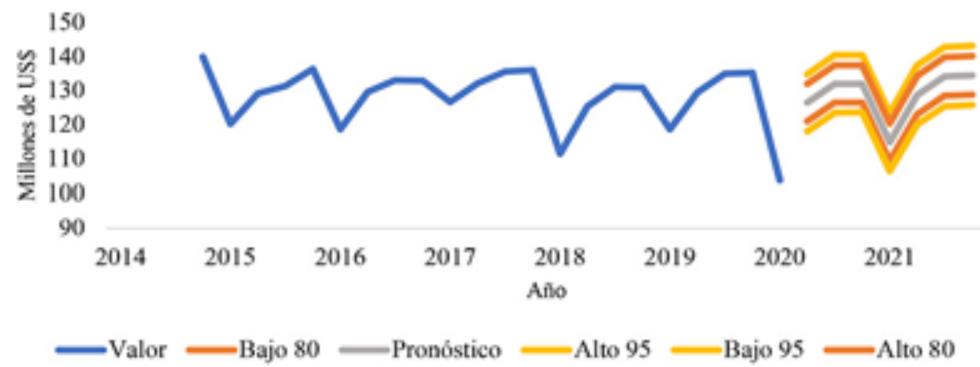

Fuente: Elaboración propia. 
Para el caso de Panamá el modelo que mejor se relaciona a los datos es el modelo ARIMA $(1,1,1)$.

Tabla No. 8 Remesas familiares en Panamá pronóstico acuerdo A MODELO ARIMA $(1,1,1)$

- Millones de US\$ -

\begin{tabular}{|l|c|c|c|c|c|}
\cline { 2 - 6 } \multicolumn{1}{c|}{} & $\begin{array}{c}\text { Promedio del } \\
\text { Pronóstico de } \\
\text { Remesas }\end{array}$ & $\begin{array}{c}\text { Pronóstico } \\
\text { Bajo con 80\% } \\
\text { de Confianza }\end{array}$ & $\begin{array}{c}\text { Pronostico } \\
\text { Alto con 80\% } \\
\text { de Confianza }\end{array}$ & $\begin{array}{c}\text { Pronostico } \\
\text { Bajo con 95\% } \\
\text { de Confianza }\end{array}$ & $\begin{array}{c}\text { Pronostico } \\
\text { Alto con 95\% } \\
\text { de Confianza }\end{array}$ \\
\hline I Trim. 2020 & 122.5 & 110.8 & 134.1 & 104.7 & 140.2 \\
\hline II Trim.2020 & 123.7 & 111.9 & 135.6 & 105.7 & 141.8 \\
\hline III Trim. 2020 & 123.4 & 110.8 & 136.1 & 104.1 & 142.8 \\
\hline IV Trim. 2020 & 123.5 & 110.3 & 136.7 & 103.3 & 143.7 \\
\hline I Trim. 2021 & 123.5 & 109.7 & 137.3 & 102.3 & 144.6 \\
\hline II Trim.2021 & 123.5 & 109.1 & 137.9 & 101.5 & 145.5 \\
\hline III Trim. 2021 & 123.5 & 108.6 & 138.4 & 100.6 & 146.4 \\
\hline IV Trim. 2021 & 123.5 & 108.0 & 139.0 & 99.8 & 147.2 \\
\hline
\end{tabular}

Fuente: Elaboración propia.

Gráfico No. 7 Pronóstico remesas familiares en Panamá.

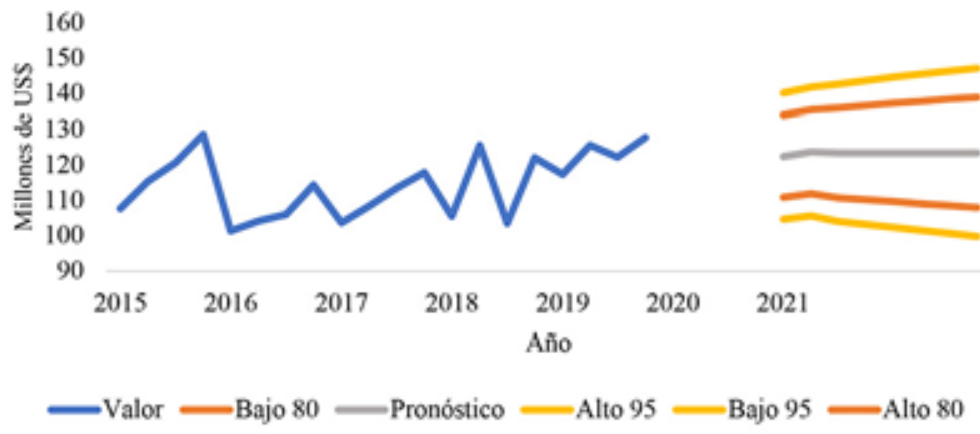

Fuente: Elaboración propia . 
Tabla No. 9 Margen de error en los pronósticos año 2020.

\begin{tabular}{|l|c|c|c|c|}
\hline \multicolumn{1}{|c|}{ País } & Valor Real & $\begin{array}{c}\text { Valor } \\
\text { Pronosticado } \\
\text { (Promedio) }\end{array}$ & $\begin{array}{c}\text { \% Margen de } \\
\text { Error de la } \\
\text { diferencia }\end{array}$ & $\begin{array}{c}\text { Margen de } \\
\text { Error en } \\
\text { Millones de } \\
\text { US\$ (+/-) }\end{array}$ \\
\hline Guatemala (agosto 2020) & $1,050.2$ & $1,047.5$ & 0.25 & 2.64 \\
\hline El Salvador (agosto 2020) & 559.3 & 496.3 & 11.27 & 63.04 \\
\hline Honduras (Julio 2020) & 520.8 & 512.1 & 1.66 & 8.67 \\
\hline Rep. Dominicana (agosto 2020) & 769.9 & 615.4 & 20.07 & 154.52 \\
\hline Panamá (I Trim. 2020) & 112.6 & 122.5 & 8.76 & -9.86 \\
\hline
\end{tabular}

Fuente: Elaboración propia.

$\mathrm{Al}$ analizar los valores reales de las remesas para los países con datos actualizados dentro del Consejo Monetario Centroamericano (2020). Considerando el valor promedio de los pronósticos, reflejan márgenes de error bajos en términos de porcentajes y en términos monetarios. El promedio del margen de error para los países de la Tabla No. 9 fue del $8.40 \%$. Lo que se traduce en márgenes apropiados que permiten reducir la incertidumbre incluso en momentos de crisis. Es necesario señalar que en el caso de Costa Rica y Nicaragua al momento de realizar el análisis respectivo no se tenían valores reales oficialmente publicados para poder comparar el margen de error con el pronóstico del modelo.

\section{Conclusiones}

La importancia de las remesas familiares es disímil en la región centroamericana de acuerdo con el volumen que de dólares que se envía a cada país, pero más homogénea de acuerdo con su importancia relativa tanto en la generación de divisas como en función del porcentaje que representa dentro de las economías (en promedio cercana al 10\% de PIB regional). Esto evidencia la importancia de contar con métodos de pronóstico adecuados para mitigar los efectos que sean realmente producto de la variable per se y aquellos más distorsionadores que se deriven políticas económicas 
basadas más en la especulación o en la presión de otros agentes con mayores asimetrías de información.

El comportamiento de las remesas familiares es una muestra de resiliencia que evidencia el compromiso económico de los centroamericanos en Estados Unidos (principalmente) con su núcleo de origen y que hacen un llamado a mitigar la formulación de escenarios poco fundamentados que incluso hablaban de un crecimiento negativo en el flujo receptor al inicio de la pandemia, aumentando la especulación en las economías. Por el contrario, países como Guatemala, El Salvador y Honduras han experimentado más bien una aceleración en los flujos enviados para cubrir los efectos nocivos de la pandemia y como medida de resguardo ante el escenario electoral en Estados Unidos, siendo este un factor coincidente con las dudas sobre un posible repunte de la enfermedad.

Particularmente en el caso de los modelos de series temporales aplicando la metodología ARIMA y SARIMA presentan un acercamiento con bajos niveles de error en los datos macroeconómicos logrando una estabilidad de los mismos. Sin embargo, es necesario la actualización constante del modelo per se, así como la actualización con datos reales, los cuales hacen que el modelo se perfeccione constantemente logrando que las series temporales tengan cada vez mejores pronósticos.

La aplicación de métodos de pronóstico efectivos y eficientes como en el caso de las remesas, puede facilitar la formulación de políticas integradoras viables y acordes con la realidad de la región, normalmente entendida como un espacio geográfico con problemáticas comunes, pero a la vez, como una oportunidad de resaltar aquellas muestras de respuesta oportuna que, para el caso de las remesas, muestran mes a mes una luz de optimismo, del compromiso y de la esperanza (más allá del COVID-19) por parte de la población centroamericana que se encuentran lejos de su país. 
BibliografíA

ÁLVAREZ, I. (2012). Mirando al norte: Algunas tendencias de la migración latinoamericana facultad latinoamericana de ciencias sociales. https://www.flacso.org/secretaria-general/mirando-alnorte-algunas-tendencias-migraci-n-latinoamericana

BANCO MUNDIAL. (2018). Moving for Prosperity: Global Migration and Labor Markets. https://openknowledge. worldbank.org/bitstream/handle/10986/29806/211281ov.pdf BOX , G., \& JENKINS, G. M. (1978). Time Series Analysis: Forecasting and Control. San Francisco.

CONSEJO MONETARIO CENTROAMERICANO. (2020).

Sistema de información macroeconómica y financiera de la región. http://www.secmca.org/simafir.html

GONZÁlEZ CASIMIRO, M. P. (2009). Análisis de Series Temporales: Modelos ARIMA. Sarriko-On.

LAROTTA, S. P. (2019). Determinantes para la migración internacional de colombianos entre 1990-2015 a partir de un modelo gravitacional. Territorios, 41,69-100.https:// doi.org/https://doi.org/10.12804/revistas.urosario.edu.co/ territorios/a.7414

ORGANIZACIÓN INTERNACIONAL PARA LAS MIGRACIONES. (2018). Informe sobre las migraciones en el mundo 2018. https://publications.iom.int/system/files/pdf/ wmr 2018 sp.pdf

POKORNY, M. (1987). An Introduction to Econometrics. Nueva York.

VILLAVICENCIO, J. (2014). Introducción a Series de Tiempo. Puerto Rico: Instituto de estadísticas de Puerto Rico.

Fecha recepción: 15 de marzo de 2021

Fecha aceptación: 24 de mayo de 2021

Fecha versión final: 31 de mayo de 2021 